\title{
p53-induced microRNA-1246 inhibits the cell growth of human hepatocellular carcinoma cells by targeting NFIB
}

\author{
QUAN ZHANG ${ }^{1,3^{*}}$, LI-YE CAO $^{2 *}$, SHU-JIE CHENG $^{3}$, AI-MIN ZHANG $^{3}$, XIAO-SHI JIN ${ }^{3}$ and YONG LI ${ }^{1}$ \\ ${ }^{1}$ Department of Surgery, The Fourth Affiliated Hospital of Hebei Medical University, Shijiazhuang, Hebei; \\ Departments of ${ }^{2}$ Ultrasound and ${ }^{3}$ Surgery, The Affiliated Hospital of Hebei University, Baoding, Hebei, P.R. China
}

Received September 5, 2014; Accepted November 29, 2014

DOI: 10.3892/or.2015.3715

\begin{abstract}
In recent years, miR-1246 has been identified as a transcriptional target of p53 in Down syndrome and may provide a new p53-miR-1246-DYRK1A-NFAT pathway in cancer. The present study aimed to explore the role of miR-1246 in the tumorigenesis of human hepatocellular carcinoma (HCC). We found that wild-type p53 regulated the expression of miR-1246 in HCC cell lines, and alteration of miR-1246 modulated cell proliferation, colony formation ability and apoptosis. The nuclear factor I/B (NFIB), an oncogene, was identified as a direct target gene of miR-1246 using a fluorescent reporter assay. Overexpression of NFIB abolished the regulation of cell apoptosis caused by miR-1246 in HepG2 cells. This finding suggests that miR-1246 is regulated by $\mathrm{p} 53$ and suppresses the growth of human HCC by targeting NFIB. Here, we propose a new p53-miR-1246NFIB pathway in HCC.
\end{abstract}

\section{Introduction}

The p53 tumor-suppressor plays a crucially role in preventing neoplasia and tumor progression. p53 directly activates a large number of genes, which mediate numerous cellular functions that contribute to tumor suppression $(1,2)$. Furthermore, p53 may mediate the specific repression of many genes. However, the mechanisms of gene repression by p53 are not well understood and may be indirect to some extent (3).

In recent years, miRNAs have been identified as important components of the signaling cascades that mediate and regulate tumor suppression exerted by p53 (4-10). In 2007, the miR-34 families, miR-34a and miR-34b/c, were reported to be directly regulated by p53 by a number of laboratories using

Correspondence to: Dr Yong Li, Department of Surgery, The Fourth Affiliated Hospital of Hebei Medical University, No. 12 Jiankang Road, Shijiazhuang, Hebei 050011, P.R. China

E-mail: liyonghappy2014@163.com

${ }^{*}$ Contributed equally

Key words: miR-1246, NFIB, cell proliferation, HCC diverse approaches (11-16). miR-1246 has been identified as a novel p53 target miRNA (17). Notably, this miRNA regulates the expression of DYRK1A, a Down syndrome-associated kinase, which inactivates a nuclear transcriptional factor called NFAT1C by phosphorylating it and preventing it from nuclear import (18).

In the present study, we found that p53 induces the expression of miR-1246 in hepatocellular carcinoma (HCC) cell lines. Alteration of miR-1246 modulated cell proliferation, colony formation ability and apoptosis. These functions were, at least in part, exerted through the direct targeting of nuclear factor I/B (NFIB). In conclusion, we propose a new p53 pathway involving miR-1246-NFIB potentially critical for cancer prevention.

\section{Materials and methods}

Human liver tumor samples. Fresh frozen human HCC tissue samples and matched normal hepatocellular tissue samples were obtained from the Fourth Affiliated Hospital of Hebei Medical University. The types of all the tumors were confirmed by pathologic analysis. All the human materials were used in accordance with the policies of the Institutional Review Board of Hebei Medical University.

Cell culture and transfection. Seven human HCC cell lines (HepG2, Hep3B, Huh7, C3A, PLC, LO2 and SUN387) were maintained in MEM $\alpha$ or RPMI-1640 medium (Gibco), respectively, and supplemented with $10 \%$ fetal bovine serum (FBS), $100 \mathrm{IU} / \mathrm{ml}$ of penicillin and $100 \mu \mathrm{g} / \mathrm{ml}$ of streptomycin. Cells were incubated at $37^{\circ} \mathrm{C}$ in a humidified chamber supplemented with $5 \% \mathrm{CO}_{2}$. Transfection was performed with Lipofectamine 2000 reagent (Invitrogen, Carlsbad, CA, USA) following the manufacturer's protocol.

Fluorescent report assay. To confirm the direct interaction between miR-1246 and NFIB mRNA, HCC cells were simultaneously transfected with miR-1246 or miR-control and the reporter vectors in 48-well plates. The cells were lysed with radioimmunoprecipitation assay (RIPA) buffer $(150 \mathrm{mM}$ $\mathrm{NaCl}, 50 \mathrm{mM}$ Tris- $\mathrm{HCl}$ pH 7.2, 1\% Triton X-100, 0.1\% SDS) $72 \mathrm{~h}$ later, and the proteins were harvested. The intensities of luciferase were detected with a fluorescence spectrophotometer F-4500 (Hitachi, Tokyo, Japan). 
3-(4,5-Dimethylthiazol-2-yl)-2,5-diphenyltetrazolium bromide (MTT) assay. Cell proliferation was determined using the MTT cell proliferation kit (Solarbio, Beijing, China). HCC cells $\left(5 \times 10^{3}\right)$ were seeded in each 96 -well plate and allowed to adhere overnight. The cells were then infected with the adenovirus vector at an MOI of 10 for the indicated times prior to being used for the MTT assay as per the manufacturer's instructions (Bio-Rad Laboratories Inc., Irvine, CA, USA).

Colony formation assay. After transfection, cells were counted and seeded in 12-well plates in triplicate at 100 cells/well. Fresh culture medium was replaced every 3 days. The colony was counted only if it contained $>50$ cells, and the number of colonies was counted from day 6 after seeding. The rate of colony formation was calculated with the equation: Colony formation rate $=$ (number of colonies/number of seeded cells) x $100 \%$.

Quantitative reverse transcription- $P C R(q R T-P C R)$. mRNAs or miRNAs were reverse transcribed to generate cDNA using oligo(dT) primers or stem-loop reverse transcriptase (RT) primers (18), respectively. Then, U6 snRNA (for miRNA) or GAPDH (for mRNA) was considered as the endogenous control. Target genes and controls were treated under the same condition and analyzed by real-time RT-PCR using SYBR Premix Ex Taq ${ }^{\mathrm{TM}}$ (Takara, Dalian, China) according to the manufacturer's protocol. The primers used in the present study are listed in Table I.

Western blot analysis. Protein extracts were prepared with RIPA lysis buffer in the presence of proteinase inhibitors. Western blot analysis was performed using antibodies against NFIB (1:600; Cell Signaling Technology, Danvers, MA, USA) and GAPDH (1:400; Beijing Biosynthesis Biotechnology Co., Beijing, China). The blots were then developed using the enhanced chemiluminescence (ECL) reagent (Amersham Biosciences, USA).

Immunohistochemistry (IHC) staining. The anti-NFIB antibody (1:500; Abcam) was used for IHC using standard methods.

Flow cytometric analysis. At $48 \mathrm{~h}$ after transfection, the HepG2 cells were detached from the plates by trypsin incubation, rinsed with phosphate-buffered saline (PBS) and fixed in $70 \%(\mathrm{v} / \mathrm{v})$ ethanol. The cells were then rehydrated in PBS and incubated with RNase $(100 \mu \mathrm{g} / \mathrm{ml})$ and propidium iodide (PI) $(60 \mu \mathrm{g} / \mathrm{ml})$ (Sigma-Aldrich, St. Louis, MO, USA). Cells were analyzed using the FACSCalibur system (BD Biosciences, San Jose, CA, USA).

Statistical analysis. Statistical analysis utilized the two-tailed Student's t-test. Statistical significance was set as $\mathrm{p}<0.05$.

\section{Results}

p53 induces the expression of miR-1246 in HCC cell lines. We enforced the expression of p53 in HepG2 or Hep-3B cells and found that they both had induced expression of miR-1246 (Fig. 1A and B). In contrast, the knockdown of p53 abrogated the induction of miR-1246 expression in the two cell lines (Fig. 1A and B). In addition, we detected the expression of miR-1246 and p53 in $26 \mathrm{HCC}$ tumor tissues, and found that miR-1246 was consistent with the p53 expression (Fig. 1C). Next, we detected the miR-1246 expression level in $6 \mathrm{HCC}$ cell lines (two of them expressed wild-type p53, one of them expressed null p53, and three of them expressed mutant p53). The finding also suggested that the expression of miR-1246 was consistent with p53 (Fig. 1D).

Alteration of miR-1246 affects the cell growth of HCC in vitro. To further explore the role of miR-1246 in $\mathrm{HCC}$, we transfected either miR-1246 mimics or miR-1246 antisense oligonucleotides (miR-1246 ASO) into HCC cells. As shown in Fig. 1E and F, miR-1246 expression was increased $~ 8.0$ fold in the Hep-3B cells when the miR-1246 mimics were induced, while, miR-1246 ASO resulted in $\sim 74 \%$ reduction in miR-1246 levels in the HepG2 cells. Cell viability of HCC cells transfected with miR-1246 or miR-1246 ASO was evaluated by the MTT assay. miR-1246 reduced cell viability at 36 or $48 \mathrm{~h}$ after transfection, whereas miR-1246 ASO increased HCC cell viability (Fig. 1G and I). In parallel, we analyzed colony formation and cellular proliferation to assess the effect of miR-1246 on the proliferative capacity of HCC cells. Compared with the control group, transfection with miR-1246 was $\sim 50 \%$ less than that of Hep3B cells transfected with the miR-control (Fig. 1H). Conversely, the colony formation rate of the HepG2 cells after transfection with miR-1246 ASO was increased 2.3-fold when compared with the control group (Fig. 1J). These results indicate that miR-1246 inhibits the cell proliferation of HCC cells.

miR-1246 targets NFIB and negatively regulates its expression. miR-1246 has been demonstrated to play an essential role in the regulation of growth and apoptosis of cells (17-21). To determine the mechanism of miR-1246-mediated cell proliferation and apoptosis regulation in HCC cells, we next identified target genes that could be responsible for the effect of miR-1246. The oncogene NFIB, which was predicted to have two miR-1246 binding sites in its 3'-untranslated region (3'UTR) (Fig. 2A), was chosen for further study. To confirm whether miR-1246 could bind to this predicted region and suppress the protein expression of NFIB, we constructed a luciferase reporter vector in which the 3'UTR fragment of NFIB, including the putative binding site, was inserted downstream of the luciferase coding region. HepG2 cells were transfected with the reporter vector together with either miR-1246 ASO or miR-1246. As shown in Fig. 2B, the intensity of luciferase was higher in the miR-1246-blocked group as compared to the control group, whereas ectopic expression of miR-1246 decreased the intensity of luciferase intensity compared with the control group. In addition, we constructed two other luciferase reporter vectors containing mutations in the miR-1246 binding sites (Fig. 2A). Neither blocking miR-1246 with ASO nor overexpressing miR-1246 had any effect on the intensity of fluorescence from the vector with the mutated miR-1246 binding region (Fig. 2B). These results showed that miR-1246 binds directly to the 3'UTR of NFIB.

To determine whether miR-1246 negatively regulates NFIB expression at the mRNA or protein level, we assessed 
Table I. Primers and sequences used in the present study.

\begin{tabular}{lll}
\hline Name & \multicolumn{1}{c}{ Primer } & \multicolumn{1}{c}{ Sequence } \\
\hline U6 & Forward & 5'-GTGCTCGCTTCGGCAGCACATATAC-3' \\
& Reverse & 5'-AAAAATATGGAACGCTCACGAATTTG-3' \\
GAPDH & Forward & 5'-ATGTCGTGGAGTCTACTGGC-3' \\
& Reverse & 5'-TGACCTTGCCCACAGCCTTG-3' \\
miR-1246 & Forward & 5'-TATTGCACTCGTCCCCTGCT-3' \\
& Reverse & 5'-GTGCAGGGTCCGAGGT-3' \\
NFIB & Forward & 5'-AAACCCAGCACTTTGTGTCC-3' \\
& Reverse & 5'-TCTTGGGGAAGAATCCTGTG-3' \\
miR-1246 ASO & Antisense miR-1246 & 2'-O-Me-CCTGCTCCAAAAATCCTTT
\end{tabular}
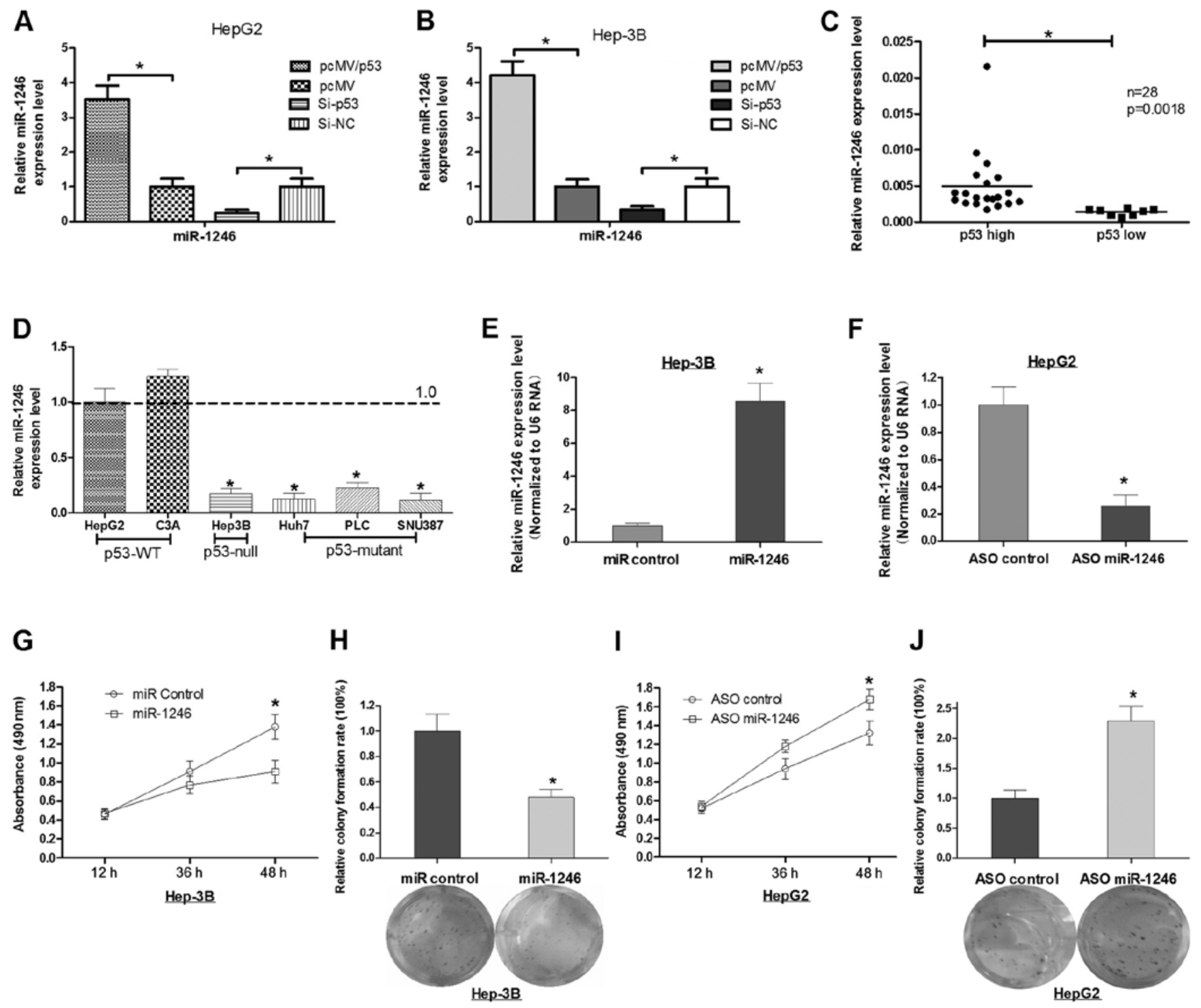

Figure 1. (A and B) The relative miR-1246 expression level after overexpression and knockdown of p53 in HepG2 cells. (C and D) The relative miR-1246 expression level in $28 \mathrm{HCC}$ tissues and $6 \mathrm{HCC}$ cell lines. The expression level of miR-1246 in HCC cells was detected by real-time RT-PCR and normalized to U6 snRNA. ("P<0.05). (E and F) Alteration of miR-1246 levels affects the growth of HCC cells. The miR-1246 expression level in HCC cells was effectively altered by transfection with miR-1246 ASO or miR-1246 as detected by real-time RT-PCR. U6 snRNA was used for normalization. (G and I) MTT assay was used to determine relative cellular proliferation at 36 and $48 \mathrm{~h}$. A490 is the optical density of MTT measured at $490 \mathrm{~nm}$. The data at 36 and $48 \mathrm{~h}$ after transfection with miR-1246 or miR-1246 ASO showed a statistically significant difference. (H and J) Cell growth was measured via colony formation assays and proliferation curve assays. 
A putative site1

NFIB-3'UTR WT 5' ACAAAGAGGCAGGCAAAUCCAUG miR-1246 3' GGACGAGGUUUUUAGGUA

NFIB-3'UTR mut1 5'..ACAAAGAGGCAGGC ECCGACC \$.. putative site2

miR-1246 3' GGACGAGGUUUUUAGGUAA

NFIB-3'UTR mut2 5 '..UUCUUGUAUUGACAG ECACCAG..

C

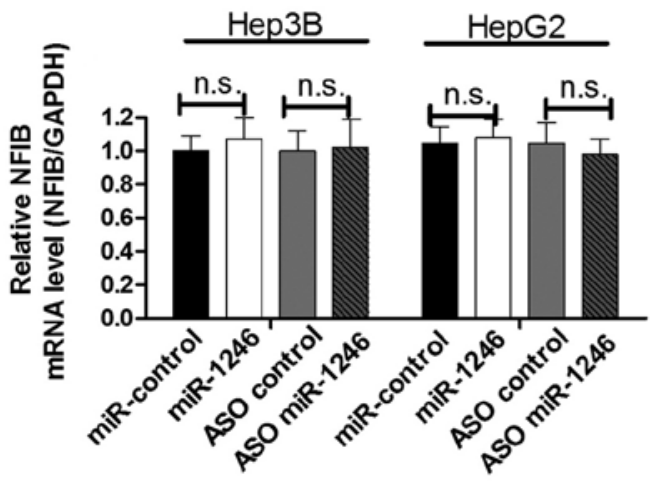

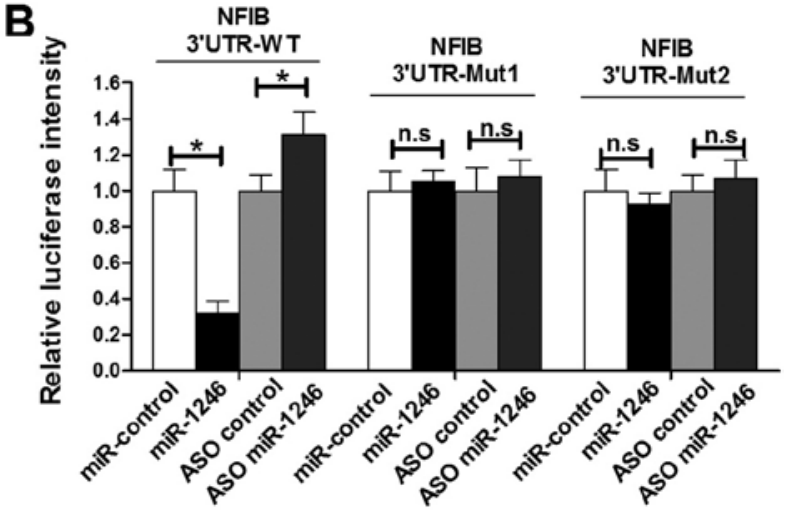

D
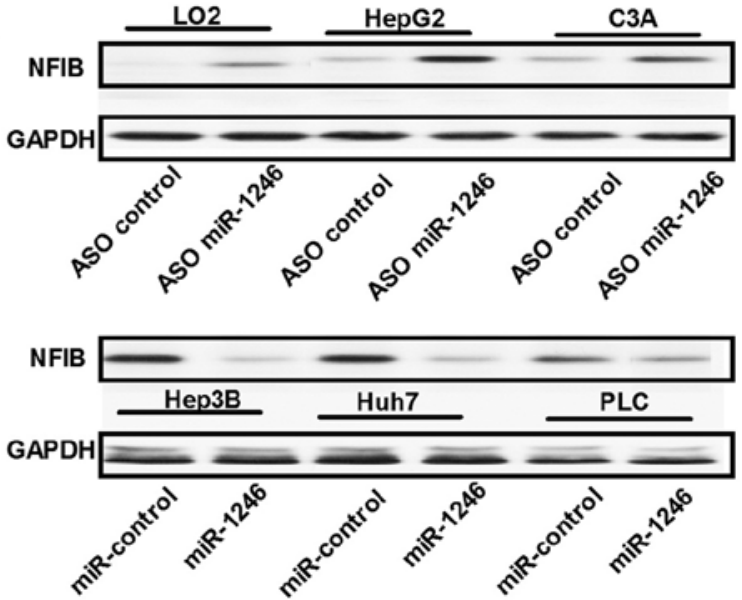

Figure 2. NFIB is a direct target of miR-1246. (A) The NFIB 3'UTR carries two potential miR-1246 binding sites. The direct interaction of miR-1246 and NFIB mRNA was confirmed by a luciferase reporter assay. (B) HepG2 cells were transfected with a luciferase reporter vector together with miR-1246 ASO or miR-1246, and the luciferase intensity was measured. The data represent the means \pm SD of three different experiments ("P<0.05). (C) RT-PCR was used to detect the expression level of NFIB mRNA. miR-1246 was unable to alter the mRNA level of NFIB, respectively, as compared to the control group. (D) When miR-1246 was blocked or overexpressed, the protein level of NFIB in the HCC cells was subsequently elevated or diminished, respectively, as compared to the control group ( $(\mathrm{P}<0.05)$. n.s. nonsignificant; NFIB, nuclear factor I/B.

endogenous NFIB expression in HepG2 or Hep3B cells with altered miR-1246 expression. Both cell lines were transfected with miR-1246 or miR-1246 ASO to overexpress or block miR-1246, respectively. Notably, miR-1246 showed an inability to alter NFIB expression at the mRNA level in both cell groups transfected with miR-1246 or miR-1246 ASO (Fig. 2C). However, miR-1246 downregulated NFIB protein expression in the $\mathrm{LO} 2, \mathrm{HepG} 2$ and $\mathrm{C} 3 \mathrm{~A}$ cells, while miR-1246 ASO induced increased NFIB protein expression in the Hep-3B, Huh7 and PLC cells (Fig. 2D). These results suggest that miR-1246 regulates endogenous NFIB expression by targeting mRNAs and triggering translation repression.

Knockdown of NFIB inhibits HCC cell growth and promotes its apoptosis. Sequence-specific small interfering RNAs (siRNAs) can effectively suppress gene expression. An siRNA targeting NFIB (siR-NFIB) was used for the present study. Western blot assay showed that the level of NFIB expression was reduced by $\sim 90 \%$ in the HepG 2 cells that were transfected with siR-NFIB, as compared to HepG2 cells transfected with a control siRNA (Fig. 3A). Inhibition of NFIB expression inhibited cell growth and increased HepG2 cell apoptosis as compared to the control group (Fig. 3B-D), which was consistent with the effects of miR-1246 overexpression.
Overexpression of NFIB abolishes the regulation of cell apoptosis caused by miR-1246. To further explore the miR-1246-induced HCC cell proliferation inhibition mediated by NFIB, we transfected HepG2 cells with the miRcontrol, miR-1246 or miR-1246/NFIB expression plasmid (pcDNA3/NFIB), respectively. Western blotting confirmed that the NFIB expression vector was effective (Fig. 3E). Based on the results, ectopic expression of NFIB reversed the effects of miR-1246 on HCC cell phenotypes (Fig. 3F-J). These results revealed that NFIB was a key mediator in the miR-1246-induced cell growth inhibition in HCC.

NFIB is inversely expressed with miR-1246 in HCC. To explore the relevance of NFIB in relation to miR-1246 in human HCC cells, the expression of NFIB was evaluated in HCC cells by western blotting using the anti-NFIB antibody. The expression of NFIB was increased in all mutant or null p53-containing HCC cell lines with p53 wild-type cells, which was inversely correlated with miR-1246 (Fig. 4A and B). In addition, IHC staining showed predominantly cytoplasmic localization of NFIB, and the expression of NFIB was markedly increased in all malignant tumor cells from the examined archival HCC samples relative to the adjacent non-tumor tissues (Fig. 4C and D). In total, we found that the expres- 

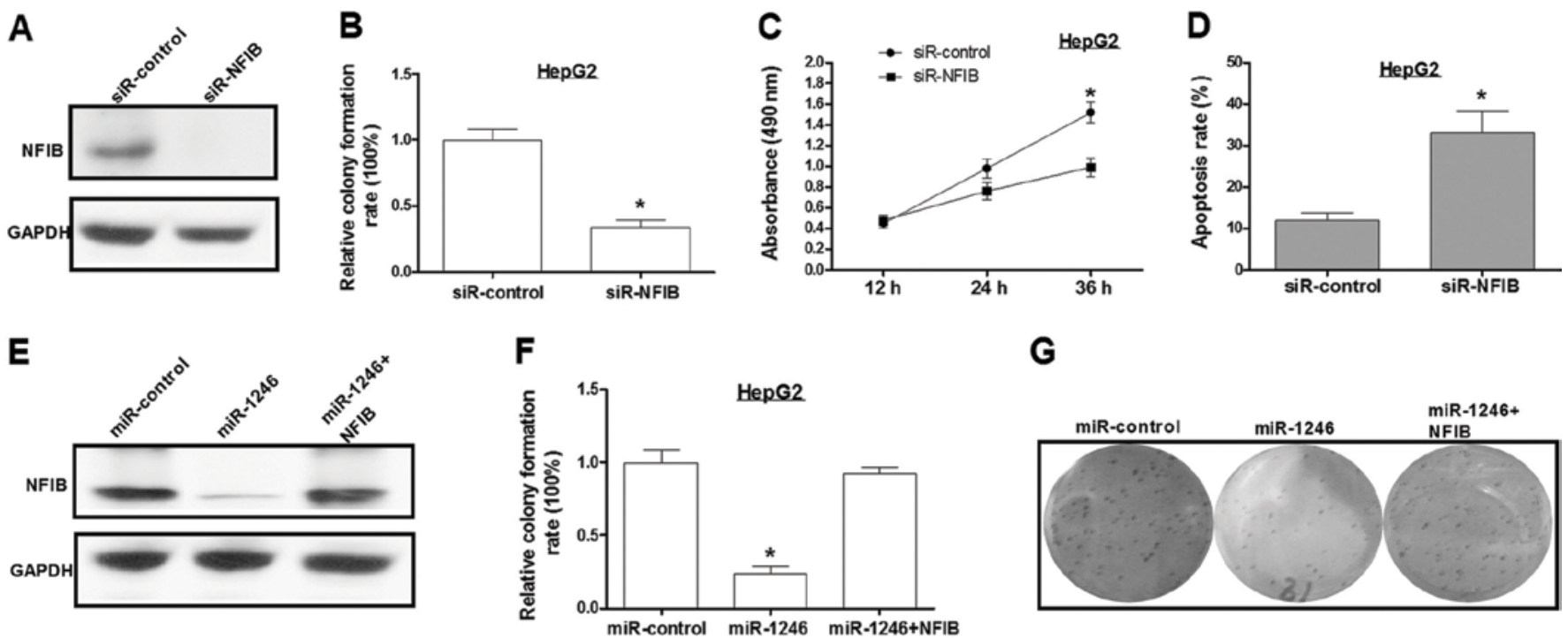

G
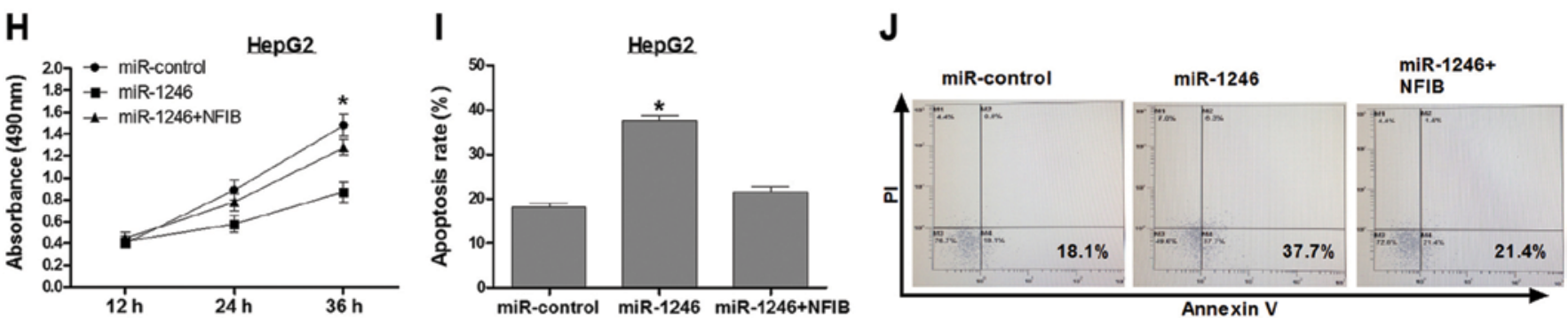

Figure 3. Knockdown of NFIB shows concordant effects with miR-1246 overexpression in HCC cells. (A) Western blot analysis showed that the expression of NFIB was successfully suppressed by NFIB siRNA. NFIB was knocked down in HCC cells, and cell growth/viability activity was analyzed with (B) colony formation and (C) MTT assays. (D) Apoptosis was analyzed by FACS. The data represent the means \pm SD of three different experiments ("P $<0.05$ ). HCC cells were transfected with miR-1246, pcDNA3/NFIB or miRNA control. Overexpression of NFIB abolished the regulation of cell apoptosis caused by miR-1246. (E) At $48 \mathrm{~h}$ after transfection, NFIB expression was measured by western blotting. Cell growth/viability was analyzed with (H) MTT, (F and G) colony formation and ( $\mathrm{I}$ and $\mathrm{J}$ ) apoptosis assays. The data represent the means $\pm \mathrm{SD}$ of three different experiments ( $\mathrm{P}<0.05)$. NFIB, nuclear factor $\mathrm{I} / \mathrm{B}$.

sion of miR-1246 could be induced by wild-type p53 in the HCC cell lines. Overexpression of miR-1246 inhibited the proliferation of HCC cells by targeting NFIB. In contrast, the expression of miR-1246 was reduced in the mutant or null p53 HCC cells, and downregulation of miR-1246 resulted in upregulation of NFIB, subsequently promoting $\mathrm{HCC}$ cell proliferation (Fig. 4E).

\section{Discussion}

Hepatocellular carcinoma (HCC) is one of the most common types of cancers, and ranks as the fifth most leading cause of cancer-related deaths worldwide (22). Deregulated miRNA expression has been closely associated with the abnormal expression of cellular genes that regulate apoptosis, proliferation, the cell cycle and metastasis, leading to the development and progression of HCC (23-26). It has been reported that miR-1246 is a p53 target miRNA, as p53 inhibited DYRK1A expression through the induction of miR-1246 in non-small cell lung cancer cells (18). We aimed to ascertain whether miR-1246 is also induced by $\mathrm{p} 53$ and plays a role as a tumor suppressor in HCC cells. To address this issue, we first examined miR-1246 expression after overexpressing or the knockdown of p53 in HCC cells by real-time RT-PCR, as previously described (27). We then examined miR-1246 expression in p53 wild-type, mutant and null p53-containing HCC cell lines. The results showed that the expression of miR-1246 was correlated with the expression of wild-type p53. Then, we determined the effect of miR-1246 on HCC cells by gain- and loss-of-function approaches. MTT and colony formation assays showed that miR-1246 inhibited the growth of HCC cells. Thus, we inferred that miR-1246 may be a growth-inhibition factor in HCC.

The fundamental function of miRNAs is to regulate their targets by direct cleavage of mRNAs or by inhibition of translation (18), depending on the degree of complementarity with the 3'UTR of their target genes. The putative targets of miR-1246 were predicted using the miRanda, PicTar and TargetScan target algorithms, which may be correlated with the phenotype of the HCC cell lines caused by the alteration of miR-1246. Among them, the oncogene nuclear factor I/B (NFIB) was identified as a novel direct target of miR-1246. qRT-PCR and western blot assay showed that miR-1246 decreased the NFIB expression at the protein but not the mRNA level in the HCC cell lines compared with the control. In addition, NFIB expression was upregulated in the HCC tissues compared to that in the normal tissues. We confirmed that miR-1246 can bind directly to the NFIB 3'UTR and negatively regulates NFIB gene expression by a luciferase reporter assay. We then confirmed the oncogenic role of NFIB in the HCC cells, and found that knockdown of NFIB by siRNA promoted 
A

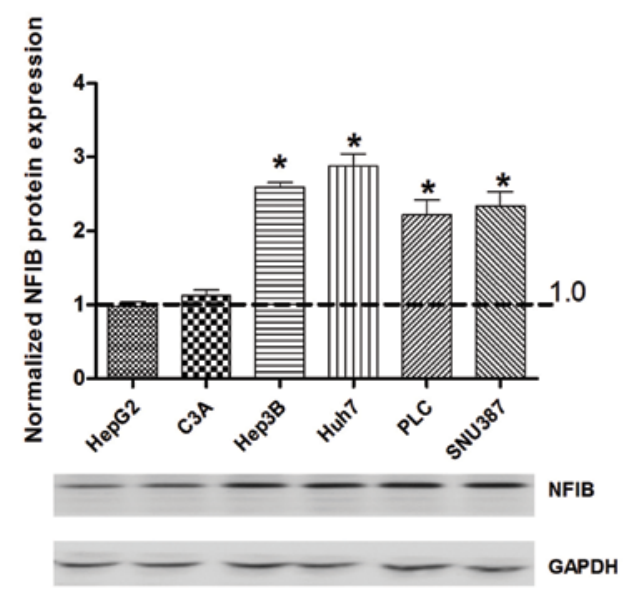

B

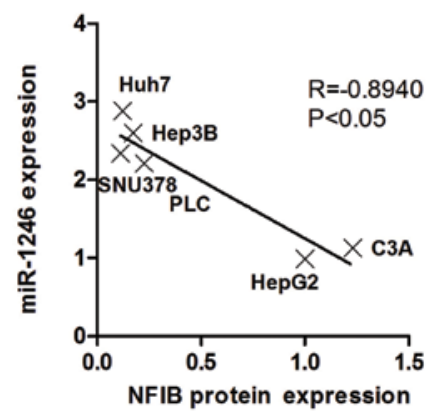

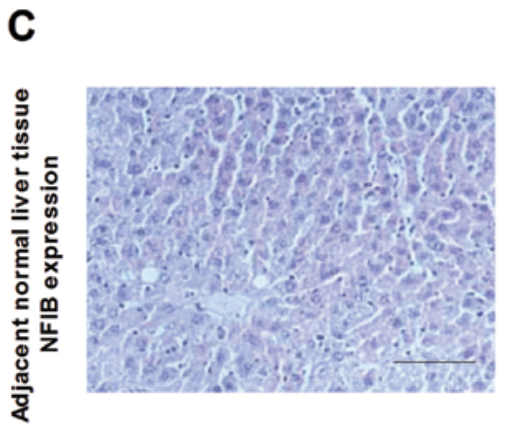

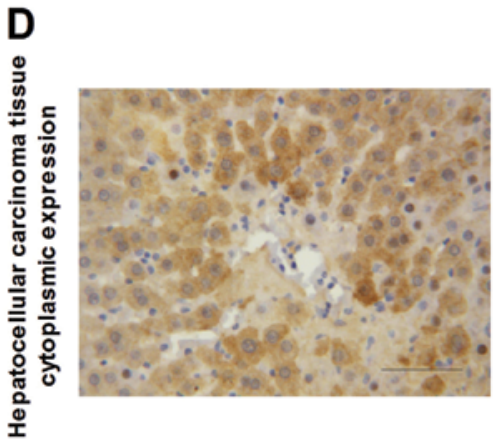

$\mathbf{E}$

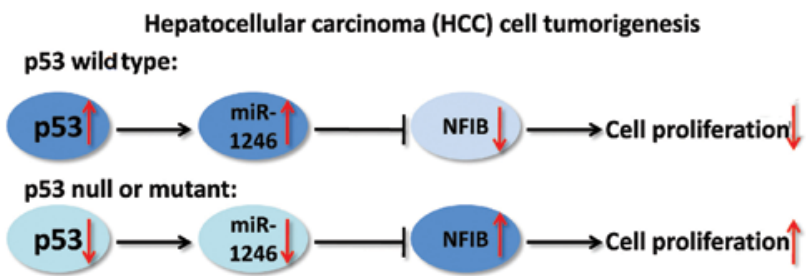

Figure 4. (A) Expression of NFIB in HCC cells as detected by western blotting. (B) Relationship between the expression of NFIB and miR-1246 was analysis by GraphPad 5.0. (C and D) Representative IHC images of NFIB protein expression. Images representing (C) negative (-) NFIB expression and (D) a high level $(+++)$ of NFIB expression. Strong IHC staining was noted in the cytoplasm and perinuclear localization was observed. (E) A model documenting the function of p53/miR-1246/NFIB in HCC tumorigenesis. NFIB, nuclear factor I/B.

HCC cell apoptosis, whereas ectopic expression of NFIB effectively alleviated the miR-1246-induced apoptosis of HCC cells. Collectively, these findings indicate that miR-1246 may exert its effects in HCC mainly by targeting NFIB. However, Chen et al recently reported that miR-1246 promotes $\mathrm{SiHa}$ cervical cancer cell proliferation, invasion and migration through suppression of its target gene thrombospondin 2 (20), which is paradoxical with our results in HCC. One possible explanation is that miRNAs have different functions in different types of tissues; for instance, miR-9 is upregulated in breast cancer cells (28), yet downregulated in human ovarian cancer (29).

Recent studies found that NFIB regulates cell viability and proliferation during transformation, and is an oncogene in small cell lung cancer (30). Furthermore, silencing of the NFIB gene resulted in reduced proliferation and an increase in the apoptotic signaling pathway, suggesting a potential value of NFIB as a novel target in ER-negative breast cancer (31). These results are consistent with our studies in HCC cells.

In conclusion, we found that p53 induced the expression of miR-1246 in HCC cell lines. Overexpression of miR-1246 promoted the cell apoptosis of HCC cells possibly through inhibition of NFIB. Thus, identification of miR-1246 and its target gene NFIB may help us to understand the molecular mechanism of tumorigenesis in HCC and may propose a new p53-miR-1246-NFIB pathway in HCC cancer.

\section{References}

1. Collavin L, Lunardi A and Del Sal G: p53-family proteins and their regulators: hubs and spokes in tumor suppression. Cell Death Differ 17: 901-911, 2010.

2. Qian Y and Chen X: Tumor suppression by p53: making cells senescent. Histol Histopathol 25: 515-526, 2010.

3. Menendez D, Inga A and Resnick MA: The expanding universe of p53 targets. Nat Rev Cancer 9: 724-737, 2009. 
4. Hermeking H: The $m i R$-34 family in cancer and apoptosis. Cell Death Differ 17: 193-199, 2010.

5. Hermeking H: MicroRNAs in the p53 network: micromanagement of tumour suppression. Nat Rev Cancer 12: 613-626, 2012.

6. Shin S, Cha HJ, Lee EM, et al: MicroRNAs are significantly influenced by p53 and radiation in HCT116 human colon carcinoma cells. Int J Oncol 34: 1645-1652, 2009.

7. He L, He X, Lowe SW and Hannon GJ: microRNAs join the p53 network - another piece in the tumour-suppression puzzle. Nat Rev Cancer 7: 819-822, 2007.

8. Feng Z, Zhang $\mathrm{C}$, Wu R and $\mathrm{Hu} \mathrm{W}$ : Tumor suppressor p53 meets microRNAs. J Mol Cell Biol 3: 44-50, 2011.

9. Zhang W and Cohen SM: The Hippo pathway acts via p53 and microRNAs to control proliferation and proapoptotic gene expression during tissue growth. Biol Open 2: 822-828, 2013.

10. Liao JM, Cao B, Zhou X and Lu H: New insights into p53 functions through its target microRNAs. J Mol Cell Biol 6 : 206-213, 2014.

11. Tarasov V, Jung $\mathrm{P}$, Verdoodt $\mathrm{B}$, et al: Differential regulation of microRNAs by p53 revealed by massively parallel sequencing: miR-34a is a p53 target that induces apoptosis and G1-arrest. Cell Cycle 6: 1586-1593, 2007.

12. Chang TC, Wentzel EA, Kent OA, et al: Transactivation of miR-34a by 53 broadly influences gene expression and promotes apoptosis. Mol Cell 26: 745-752, 2007.

13. Raver-Shapira N, Marciano E, Meiri E, et al: Transcriptional activation of miR-34a contributes to p53-mediated apoptosis. Mol Cell 26: 731-743, 2007.

14. Bommer GT, Gerin I, Feng Y, et al: p53-mediated activation of miRNA34 candidate tumor-suppressor genes. Curr Biol 17: 1298-1307, 2007.

15. Corney DC, Flesken-Nikitin A, Godwin AK, Wang W and Nikitin AY: MicroRNA-34b and microRNA-34c are targets of p53 and cooperate in control of cell proliferation and adhesionindependent growth. Cancer Res 67: 8433-8438, 2007.

16. He L, He X, Lim LP, et al: A microRNA component of the p53 tumour suppressor network. Nature 447: 1130-1134, 2007.

17. Liao JM, Zhou X, Zhang Y and Lu H: miR-1246: a new link of the p53 family with cancer and Down syndrome. Cell Cycle 11: 2624-2630, 2012.

18. Zhang Y, Liao JM, Zeng SX and Lu H: p53 downregulates Down syndrome-associated DYRK1A through miR-1246. EMBO Rep 12: 811-817, 2011.
19. Li W, Wu YF, Xu RH, Lu H, Hu C and Qian H: miR-1246 releases RTKN2-dependent resistance to UVB-induced apoptosis in HaCaT cells. Mol Cell Biochem 394: 299-306, 2014.

20. Chen J, Yao D, Zhao S, et al: MiR-1246 promotes SiHa cervical cancer cell proliferation, invasion, and migration through suppression of its target gene thrombospondin 2. Arch Gynecol Obstet 290: 725-732, 2014.

21. Xu LJ, Jiang T, Zhao W, et al: Parallel mRNA and microRNA profiling of HEV71-infected human neuroblastoma cells reveal the up-regulation of miR-1246 in association with $D L G 3$ repression. PLoS One 9: e95272, 2014.

22. Ferlay J, Shin HR, Bray F, Forman D, Mathers C and Parkin DM: Estimates of worldwide burden of cancer in 2008: GLOBOCAN 2008. Int J Cancer 127: 2893-2917, 2010.

23. Huang $S$ and $\mathrm{He} X$ : The role of microRNAs in liver cancer progression. Br J Cancer 104: 235-240, 2011.

24. Wu N, Liu X, Xu X, et al: MicroRNA-373, a new regulator of protein phosphatase 6 , functions as an oncogene in hepatocellular carcinoma. FEBS J 278: 2044-2054, 2011.

25. Zhou L, Yang ZX, Song WJ, et al: MicroRNA-21 regulates the migration and invasion of a stem-like population in hepatocellular carcinoma. Int J Oncol 43: 661-669, 2013.

26. Zhou J,Lu S, Yang S, et al: MicroRNA-127 post-transcriptionally downregulates Sept7 and suppresses cell growth in hepatocellular carcinoma cells. Cell Physiol Biochem 33: 1537-1546, 2014.

27. Chen C, Ridzon DA, Broomer AJ, et al: Real-time quantification of microRNAs by stem-loop RT-PCR. Nucleic Acids Res 33: e179, 2005

28. Ma L, Young J, Prabhala H, et al: miR-9, a MYC/MYCN-activated microRNA, regulates E-cadherin and cancer metastasis. Nat Cell Biol 12: 247-256, 2010.

29. Tang H, Yao L, Tao X, et al: miR-9 functions as a tumor suppressor in ovarian serous carcinoma by targeting TLN1. Int J Mol Med 32: 381-388, 2013.

30. Dooley AL, Winslow MM, Chiang DY, et al: Nuclear factor I/B is an oncogene in small cell lung cancer. Genes Dev 25: 1470-1475, 2011.

31. Moon HG, Hwang KT, Kim JA, et al: NFIB is a potential target for estrogen receptor-negative breast cancers. Mol Oncol 5 : 538-544, 2011. 